CASE REPORT

\title{
Perianal apocrine adenocarcinoma arising in a benign apocrine adenoma; first case report and review of the literature
}

\author{
K N MacNeill, R H Riddell, D Ghazarian
}

J Clin Pathol 2005;58:217-219. doi: 10.1136/icp.2004.021394

Benign apocrine lesions have been described in the anogenital region, although according to the World Health Organisation convincing examples of anal apocrine adenocarcinomas have not been published. This report describes the case of an invasive apocrine adenocarcinoma arising in a benign adenoma in the perianal region of a 45 year old woman. The origin and invasiveness are supported by histological and immunohistochemical studies.

$\mathrm{B}$ enign apocrine lesions have been described in the anogenital region. ${ }^{1-3}$ However, according to the World Health Organisation, convincing examples of anal apocrine adenocarcinomas have not been published. ${ }^{4}$ We describe the case of an invasive apocrine adenocarcinoma arising in a benign adenoma in the perianal region of a 45 year old woman. The origin and invasiveness are supported by histological and immunohistochemical studies.

\section{CASE REPORT}

A 45 year old woman presented with a one month history of a painless, flesh coloured, $1-2 \mathrm{~cm}$ mass in the perianal region not involving the anal canal. The lesion was excised and showed the following characteristics.

At low power (fig 1A), there is a poorly circumscribed glandular lesion in the dermis. Central to the lesion is a large, dilated single excretory duct that opens directly to the surface epithelium. A branching and budding network of glands opens into the dilated dermal component of this duct. The remainder of the lesion is composed of ill formed lobules containing elongated, branching, irregular shaped glands, some cystically dilated.

Detailed examination of the lesion (fig 1B) showed that the excretory duct was lined by stratified squamous epithelium near its epidermal opening, but was lined by a double layer of cuboidal cells as it extended into the dermis. This epithelial lining was focally thrown into micropapillary projections.

A double layer of cells lined the glandular components of the lesion that empty into the duct and those forming the lobules (fig $\mathrm{lC}$ ). There was an inner layer of cuboidal cells with round to oval nuclei with a fine chromatin pattern, occasional nucleoli, and eosinophilic cytoplasm. These cells showed the characteristic decapitation secretion of apocrine cells (fig 1D). Mitoses were rare and nuclear atypia was not seen. Focally, there was multilayering of the apocrine cells. The lesion was supported by a relatively dense stroma, with a moderate inflammatory cell infiltrate composed predominantly of lymphocytes and plasma cells (fig IC, D). Scattered neutrophils were identified. The outer layer of cells was a flattened myoepithelial layer, demonstrated by positive immmunoreactivity to muscle specific actin (MSA) (fig 2A).

The lesion was periodic acid Schiff positive and was immunoreactive for cytokeratin 7, the oestrogen receptor (ER), the progesterone receptor (PR), and gross cystic disease fluid protein (fig 2B-F). Its location, morphology, and immunohistochemical profile were consistent with an apocrine lesion.

Near and extending to a resection margin, the lesion developed a malignant architecture characterised by angulated, tapering glands (fig 3A). More importantly, however, these glands were characterised by loss of the outer myoepithelial cell layer when stained for muscle specific antigen (MSA) (fig 3B), a feature consistent with malignancy.

The loss of the myoepithelial layer and the infiltrative growth pattern led to the diagnosis of a well differentiated invasive apocrine adenocarcinoma arising within a benign apocrine adenoma. Interestingly, the cells in the invasive area showed no atypia or increased mitotic activity (consistent with a well differentiated tumour), but they did show an altered immunohistochemical profile. Unlike the remainder of the lesion, the glands were negative for both ER and PR (fig 2C, D).

\section{DISCUSSION}

Apocrine glands are unique in both their histology and anatomical location. Histologically, apocrine glands are characterised by an excretory duct with a straight intradermal component lined by stratified squamous epithelium, usually opening into a hair follicle and a secretory tubular component lined by a single layer of secretory cells with eosinophilic cytoplasm surrounded by an outer layer of myoepithelial cells. The inner layer of epithelial cells is characterised by decapitation secretion, a feature considered pathognomonic for apocrine differentiation. ${ }^{125}$ Unlike eccrine glands, which are found throughout the body, apocrine glands are found only in specific anatomical sites, including the axillae and anogenital region. ${ }^{2}$

Although carcinomas arising from apocrine glands have been described in the scalp, axillae, and vulva, ${ }^{6-8}$ according to the World Health Organisation, no convincing examples of anal apocrine adenocarcinoma have been published. ${ }^{4}$ We believe that our lesion is an example of an invasive perianal apocrine adenocarcinoma arising within a benign apocrine adenoma. This is supported by its anatomical location, histology, and immunohistochemical profile.

The lesion in our patient is located in the perianal region, where apocrine glands are abundant. It is composed of intradermal tubular and cystic components lined by epithelial

Abbreviations: ER, oestrogen receptor; MSA, muscle specific actin; PR, progesterone receptor 


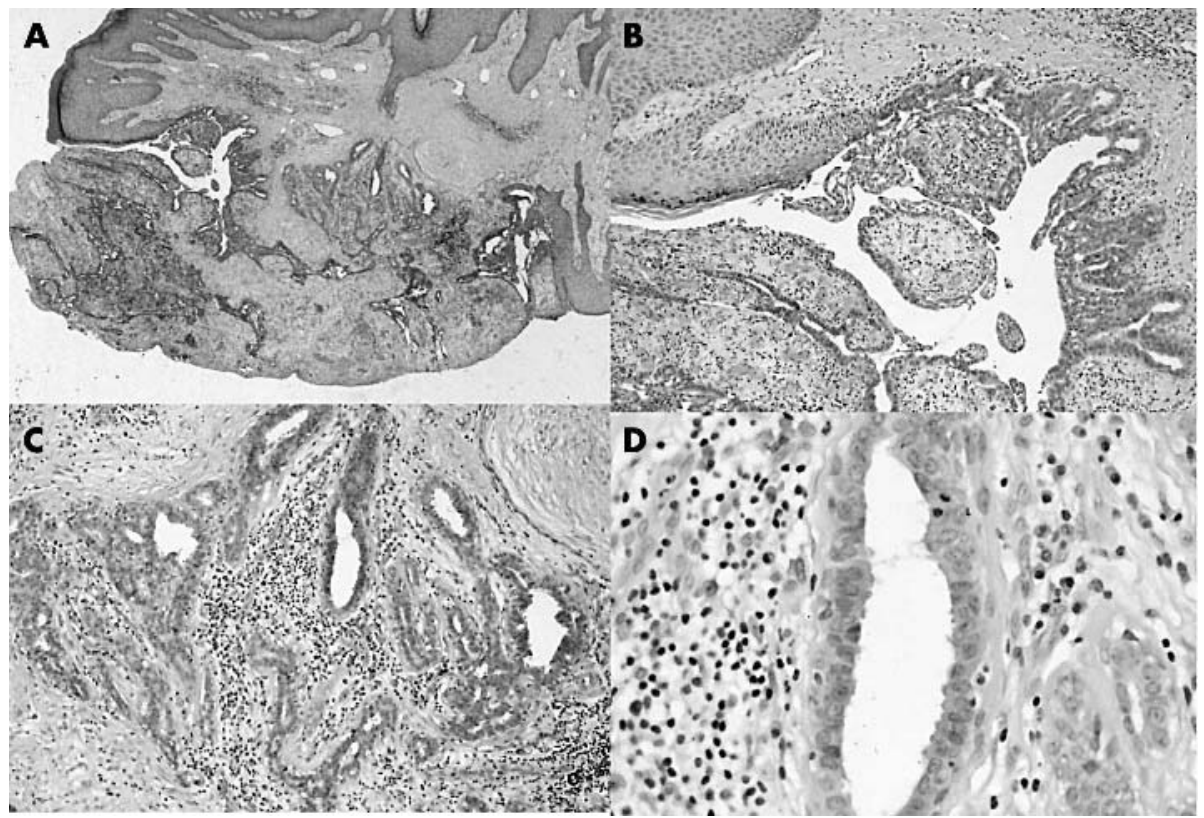

Figure 1 (A) Low power of lesion, (B) excretory duct, (C) lobular architecture, and (D) decapitation secretions.

cells, which show decapitation secretion and cytoplasmic and luminal staining for periodic acid Schiff and gross cystic disease fluid protein (fig 2E, F); features characteristic of apocrine differentiation. ${ }^{12578}$ However, the lesion described is unusual in that its excretory duct opens directly to the skin surface, rather than into a pilosebaceous unit. Although this may be uncommon, it is a recognised variation of apocrine glands. ${ }^{2}$ A similar lesion was reported in 1980 by Weigand, who described a benign "perianal apocrine adenoma" consisting of an excretory duct opening directly to the skin surface and intradermal cystic spaces, and tubules lined by cells showing decapitation secretions.

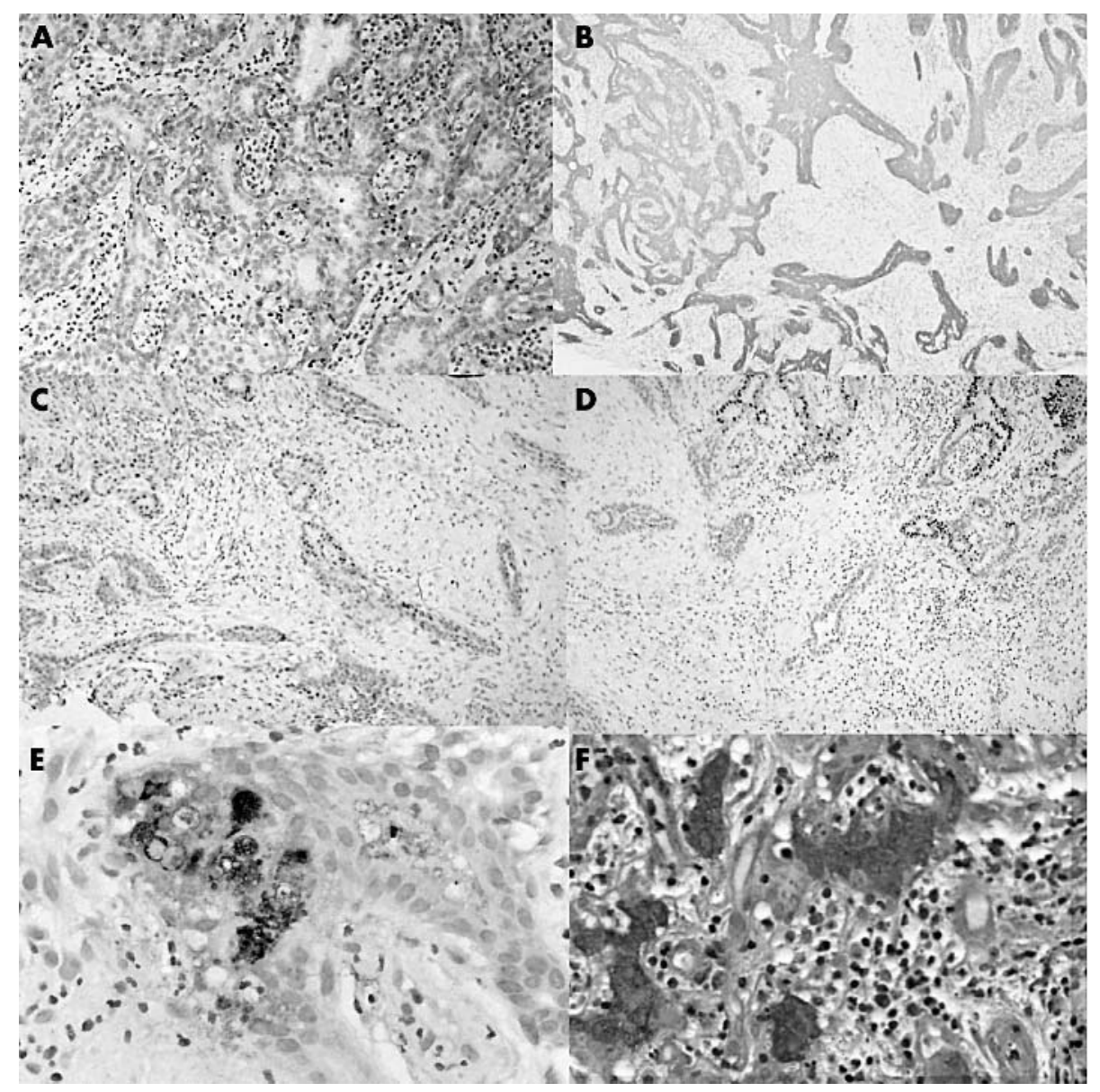

Figure 2 Immunohistochemical staining for (A) muscle specific actin; (B) cytokeratin 7; (C and D) oestrogen and progesterone receptor, respectively (note the loss of immunoreactivity in the lower lefthand corner); and (E) gross cystic disease fluid protein. (F) Staining for periodic acid Schiff. 


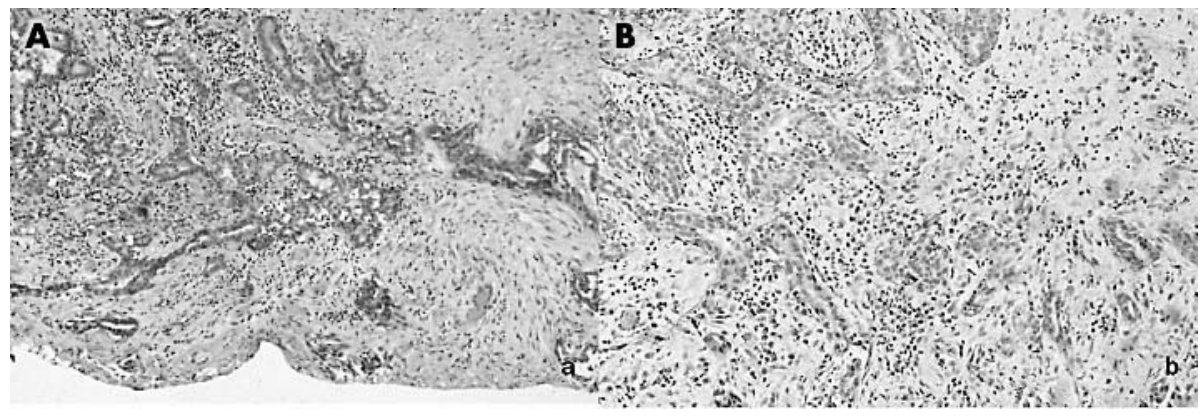

Figure 3 Invasive component

(A) Haematoxylin and eosin staining;

(B) loss of muscle specific actin

reactivity (lower right corner).

\section{Take home messages}

- This is the first convincing example of anal apocrine adenocarcinoma to be published

- The case is an invasive apocrine adenocarcinoma arising in a benign adenoma in the perianal region of a 45 year old woman

- The origin and invasiveness of this tumour are supported by the results of the histological and immunohistochemical studies

Most of the lesions described here showed cystic spaces and tubules surrounded by a myoepithelial layer, demonstrated by immunoreactivity for MSA, a feature that is characteristic of benign tissue. ${ }^{68}{ }^{9}$ However, there is a sudden loss of this myoepithelial layer, as shown by loss of MSA reactivity, in the focus of invasive adenocarcinoma (fig 3B). The loss of the myoepithelial layer is recognised as a feature of malignancy. Others have described apocrine adenocarcinomas arising from benign lesions in the vulva and axillae by demonstrating a loss of the myoepithelial layer with loss of immunoreactivity for $5100^{6}$ and smooth muscle actin. ${ }^{8}$ Nuclear pleomorphism of the epithelial cells has also been used to diagnose both invasive adenocarcinomas ${ }^{5}$ and in situ lesions. ${ }^{10}$

In contrast, the invasive component of our lesion did not show cytological atypia or an increased mitotic rate when compared with the benign areas, indicating a well differentiated adenocarcinoma. Instead, the invasive growth pattern and the loss of the myoepithelial layer establish it as a malignant lesion.

Sweat gland tumours have been shown to be immunoreactive for keratin with at least focal immunoreactivity for ER and PR. ${ }^{11}$ Pelosi et al identified focal positive immunoreactivity for ER and PR in benign apocrine ducts, but only in rare tumour cells, ${ }^{8}$ whereas Busman et al showed that apocrine carcinomas of the skin, although few in number, were not immunoreactive for ER and PR. ${ }^{12}$ These patterns support the findings of immunoreactivity in the benign portion of the lesion with loss of the immunoreactivity for ER and PR in the invasive component of our lesion.

We believe that the lesion described here is of apocrine origin because of its morphology, including decapitation secretions, and its immunohistochemical profile. The

malignancy is supported by the infiltrative pattern, the loss of the myoepithelial layer shown by loss of immunoreactivity for MSA, and non-immunoreactivity for ER and PR.

The patient underwent a subsequent wide excision, which revealed no further evidence of adenocarcinoma. She received no further treatment and at 36 months' follow up she is doing well with no evidence of recurrence.

\section{Authors' affiliations}

K N MacNeill, Department of Laboratory Medicine and Pathology, University of Toronto, Toronto, Ontario M9G 1L5, Canada

R H Riddell, Pathology and Laboratory Medicine, Mount Sinai Hospital, Toronto, Ontario M5G 1X5, Canada

D Ghazarian, Department of Pathology, University Health Network, Toronto, Ontario M5G 2M9, Canada

Correspondence to: Dr K N MacNeill, University of Toronto, Toronto, Ontario, Canada; karenmacneill@yahoo.com

Accepted for publication 25 August 2004

\section{REFERENCES}

1 Weigand DA, Burgdorf WHC. Perianal apocrine gland adenoma. Arch Dermatol 1980;116:1051-3.

2 Lever WF. Tumors of the epidermal appendages. Histopathology of the skin. Philadelphia: JB Lippincott Company, 1998.

3 Meeker JH, Neubecker RD, Helwig EB. Hidradenoma papilleferum. Am J Clin Pathol 1962;37:182-95.

4 Hamilton SR, Aaltonen LA. World Health Organisation classification of tumours. Pathology and genetics of tumours of the digestive system. Lyon: IARC Press, 2000

5 Okun MR, Finn R, Blumental G. Apocrine adenoma versus apocrine adenocarcinoma. J Am Acad Dermatol 1980;2:322-6.

6 Nishikawa Y, Tokusashi Y, Saito Y, et al. A case of apocrine adenocarcinoma associated with hamartomatous apocrine gland hyperplasia of both axillae. Am J Surg Pathol 1994; 18:832-6.

7 Paties C, Taccagni L, Papotti M, et al. Apocrine carcinoma of the skin: a clinicopathologic, immunohistochemical, and ultrastructural study. Cancer 1993;71:375-81.

8 Pelosi G, Martignoni G, Bonetti F. Intraductal carcinoma of mammary-type apocrine epithelium arising within a papillary hidradenoma of the vulva. Arch Pathol Lab Med 1991;115:1249-54.

9 Offidani A, Campanati A. Papillary hidradenoma: immunohistochemical analysis of steroid receptor profile with a focus on apocrine differentiation. J Clin Pathol 1999;52:829-32.

10 Ishida-Yamamoto A, Sato K, Wada T, et al. Syringocystadenocarcinoma papilleferum: case report and immunohistochemical comparison with its benign counterpart. J Am Acad Dermatol 2001;45:755-9.

11 Wick MR, Ockner DM, Mills SE, et al. Homologous carcinomas of the breasts, skin, and salivary glands: a histologic and immunohistochemical comparison of ductal mammary carcinoma, ductal sweat gland carcinoma, and salivary duct carcinoma. Am J Clin Pathol 1998;109:75-84.

12 Busman KJ, Tan LK, Granter SR, et al. Epidermal growth factor, estrogen and progesterone receptor expression in primary sweat gland carcinomas and primary and metastatic mammary carcinomas. Mod Pathol, 12:786-93. 